\title{
Oral Health Matters: The Ethics of Providing Oral Health During COVID-19
}

\author{
Nanette Elster ${ }^{1,2} \cdot$ Kayhan Parsi $^{1}$
}

Accepted: 11 December 2020 / Published online: 15 January 2021

(c) The Author(s), under exclusive licence to Springer Nature B.V. part of Springer Nature 2021

\begin{abstract}
Oral health is a critical part of overall health. The current COVID-19 pandemic has highlighted the importance of oral health. In this article, we describe how dental practice has been impacted by COVID-19, identify the public health response to COVID-19, and explain the gradual resumption of dental care after the initial disruption due to the pandemic. Finally, we discuss how long-standing health disparities in oral health have been exacerbated by the current pandemic.
\end{abstract}

Keywords Oral health $\cdot$ Dental care $\cdot$ Health equity $\cdot$ Bioethics $\cdot$ Pandemic . COVID-19

“You're not healthy without good oral health.” (Koop 2000)

\section{Introduction}

The current pandemic has brought ethical issues in health care to the fore with discussions of triage policies, shortages of personal protective equipment (PPE), and health care provider burn out, among many others. Conspicuously missing from the discussion, however, has been the range of ethical issues facing the dental profession and impacting oral health during the pandemic. Staffing issues, personal and community health risk, and exacerbation of health disparities are just a few of the issues that have been highlighted during the current pandemic. The importance of oral health to overall health and the dental profession's ethical responsibilities to

Nanette Elster

nelster@luc.edu

Kayhan Parsi

kparsi@luc.edu

1 Neiswanger Institute for Bioethics, Loyola University Chicago Stritch School of Medicine, 2160

S. First Avenue, Maywood, IL 60153, USA

2 Ethics Manager, American Dental Association, 211 E. Chicago Avenue, Chicago, IL 60611, USA 
serve the patient and the public at large highlight the necessity for including dentistry in any planning for, responding to, and mitigating the effects of any public health emergency.

\section{How and Why Dental Practice Has Been Impacted by COVID-19}

Dentists and their staffs are at particularly high risk of contracting COVID-19, raising questions of PPE allocation, among other salient issues. A recent infographic in the New York Times illustrates how dental health professionals are among those professionals at highest risk (Kalenderian et al. 2020). Dentists may also be at increased risk of transmitting the infection (Kalenderian et al. 2020). Additionally, unemployment among oral health professionals was notably high during March and April of 2020, with over 500,000 in the dental workforce being unemployed at that time (Kalenderian et al. 2020). These factors combined have the potential to result in increased limitations on access to oral health care. Yet, despite these setbacks, dental practices have begun reopening with adherence to new standards on safety and infection control, some of which may increase the cost of providing dental care (AHRQ 2020). This in turn may increase the cost of dental care, which for many has already been a barrier to receiving even preventive care.

These issues were exacerbated because dental care had largely fallen under the category of "non-essential" under many state and local "stay at home orders." The near shut down of all but emergency dental services for the first 4-6 weeks of the pandemic failed to recognize the significance of the essential role of dentists as primary care providers and of oral health to overall health. This exacerbated disparities among those communities already at the highest risk. With the re-opening of dental practices, the importance of preventive dental care (considered non-emergent at the outset of the pandemic) is being better understood. As this paper will discuss, dentistry, and consequently, oral health, must be part of the discussions around the ethics of pandemic planning, response and recovery. Dentists and other oral health care professionals can and should be recognized as frontline, primary care providers, and oral health care should be understood to be essential healthcare.

\section{The Initial Public Health Response to COVID-19}

By March 30, 2020, at least 30 states had stay-at-home measures to help "flatten the curve" of the highly infectious, little understood, SARS-COV-2 (COVID-19) (Mervosh et al. 2020). These orders took many different forms. In few instances did the orders rise to the level of total lockdowns as was seen in other parts of the world. In general, the orders mandated that certain businesses, such as restaurants, bars, and hair salons close down, and recommendations were made that individuals should only leave their homes for essentials, including food, medication, and necessary or urgent medical care.

In Illinois, for example, all non-essential businesses were ordered to cease operations (Pritzker 2020). Initially, even though health care was considered an essential business, all non-essential or non-emergent health care, including dentistry, 
was restricted in those states with similar orders. On March 22, however, an order from the Pennsylvania Health Department mandated that dentists cease performing services if they lacked sufficient PPE and did not have the use of a negative pressure room to reduce aerosolization (Gantz 2020). As few dental offices actually have a negative pressure room, this order essentially resulted in dentists being unable to provide even emergency care. Within days, however, after consultation with local, state and national organized dentistry, the Department of Health reversed course allowing dentists and oral surgeons to continue to provide emergency care to patients (Guggenheimer 2020a, b). The failure to recognize the nature of dental care and its critical link to healthcare more broadly was illustrated by the initial order and its subsequent, abrupt turnaround, demonstrating the importance of including oral health professionals in every facet of planning for, responding to and recovering from a public health emergency such as a pandemic.

Initially, on March 16, 2020, the American Dental Association (ADA) supported the need to limit or restrict dental procedures, recommending that all elective dental procedures be postponed, but that emergency dental care be provided in an effort to reduce volume in hospital emergency rooms (ADANews 2020). The ADA provided guidance to dentists on determining what care was considered to be a dental emergency, such as uncontrolled bleeding or trauma to facial bones that could impede breathing, and what was considered to be urgent, like severe pain, post-surgical complications, or abscess. This guidance recognized dentists' professional judgment in determining a particular patient's needs (ADA 2020). The orders to only provide emergency care, however, resulted in sparse preventive care or screening of patients. It also resulted in delays that made minor dental issues become significant dental issues. A recent study comparing dental use in March/April 2019 with March/ April 2020 showed a decrease in comprehensive oral evaluations for new or existing patients but an increase in problem-focused, more limited evaluations (FAIR Health, Inc. 2020). The ADA as well as the CDC have since developed guidance to aid in a safe return to dental practice as essential healthcare.

\section{The Gradual Resuming of Routine Dental Care}

The reopening of dental offices for more than emergency care, however, has been gradual, requiring adaptations in office set-ups, screening of staff and patients, the need for new or additional equipment and, at times, a quelling of patients' and staff members' fears. Dental offices began to re-open for routine care in May of 2020. At that time, however, the Centers for Disease Control (CDC) was still advising patients to seek dental care only in emergencies (Galewitz 2020). Even as recently as August 3, 2020, the World Health Organization was advising that nonessential dental care be delayed (WHO 2020). In the face of the ADA's strong disagreement with such a stance, the WHO offered clarification on August 12, 2020, indicating that their recommendation was only applicable in those areas with uncontrolled community transmission (Guggenheimer 2020a, b).

The national and international responses and recommendations for dental care during the pandemic have demonstrated the need to better understand how critical 
oral health is to overall health. As previously mentioned, the ADA issued a statement respectfully disagreeing with WHO. In fact, on July 27, 2020, prior to the WHO pronouncement, the Board of Trustees of the organization issued an ad interim policy on Dentistry as Essential Health Care (ADA 2020). In part, the policy states that: "Dentistry is an essential health care service because of its role in evaluating, diagnosing, preventing or treating oral diseases, which can affect systemic health" (ADA 2020).

The significance of oral health care to overall health is also recognized outside the US. In Australia, for example, the Sydney Policy Lab (2020) in their report, COVID-19 and the Dental Profession: Professional Tensions and Ethical Quandaries, stressed that:

Oral health is a key component of health, with poor oral health having significant impacts upon physical function, general health and social integration. Despite being an essential part of holistic wellbeing, dental healthcare is separated from the rest of the health system, both financially through exclusion from Medicare, and through the lack of integration of dental services with other healthcare providers. (Sidney Policy Lab 2020)

Acknowledging the significance of oral health to overall health, ensuring its delivery while the pandemic and its uncertainty persists is critical. The CDC's recently Revised Guidance for Dental Settings (2020) recognizes the significant risk/benefit calculus of providing oral health while the pandemic persists. The Guidance states:

Dental settings should balance the need to provide necessary services while minimizing risk to patients and dental healthcare personnel DHCP... [and] Provide dental treatment only after [the dentist has] assessed the patient and considered both the risk to the patient of deferring care and the risk to DHCP and patients of healthcare-associated SARS-CoV-2 transmission.

With guidance from the ADA, OSHA, and lessons learned from practitioners around the world, this is not insurmountable.

\section{Moving Forward}

The realization that oral health is part and parcel of overall health is not a new concept, but the pandemic has made glaringly clear the need to better integrate oral health care and oral health professionals into the fabric of health care and public health more generally. The Surgeon General's Report, Oral Health in America (US Department of Health and Human Services 2000) stressed this point as did the goals set forth in Healthy People (US Health and Human Services 2010).

Prior to the pandemic, access to oral health care was already a critical public health problem, but the responses taken during the pandemic have exacerbated the problem. This is reflected by the fact that there are fewer dental offices open, with many offices unable to re-tool to meet the current recommendations. Additionally, 
many of those with limited access to dental care are also those suffering from COVID-19. The following passage describes this "perfect storm" that has emerged:

It is perhaps not hyperbole to describe pandemic-related circumstances as creating a "perfect storm" in oral health care in the United States. Risk factors are elevated, access for the most vulnerable is limited, safety concerns are heightened, and the economy presents substantial challenges for patients and providers alike. The effects of COVID-19 are particularly acute for vulnerable populations, and the crisis has made evident the challenges and opportunities for oral health care in the United States. In such a time, oral health care providers and advocates must clearly communicate the importance of oral health to overall health, indicate the steps being taken to ensure patient and provider safety, and promote prevention... (Brian and Weintraub 2020)

Dentists could have and should have been part of the initial responses taken. Dentists have experience with triage, they have medical expertise and they, like other health care professionals, adhere to a social contract, having a code of ethics that directs them to not only serve individual patients but to also serve the public-at-large.

Over the past few months, dental offices have re-opened and dental practices are busy with patients. In fact, as of July, 2020, nearly $99 \%$ of dental offices have reopened (Berger 2020). One silver lining to the current pandemic is to remind us all that dentists play an important role in health care. As the Sydney Policy Lab has stated: "it is clear that the role of the dental profession extends beyond the mouth. The COVID-19 pandemic has starkly demonstrated the isolation of the dental profession which has not met its full potential through not being engaged with the wider health system" (Sydney Policy Lab 2020). The Sydney Policy is instructive for dental practice in the US. It outlines several recommendations, including making clinics suitable for health surveillance, improving teledentistry, expanding access to dental services, and developing competencies in interprofessional practice for dental students (Sydney Policy Lab 2020).

\section{COVID-19 and Oral Health Disparities}

The current pandemic has uncovered long standing health disparities based on race (Evans 2020). Disparities in dental care are particularly pernicious. Dentists working in low-income neighborhoods-who do not make as much money treating poorer patients, and who operate on thin financial margins even in good times-likely will struggle to comply, and some being forced out of business.

Meanwhile, some patients in African American and Latinx communities, already coping with high coronavirus infection rates, are anxious about undergoing closeup dental work in the middle of a pandemic. And the delayed opening of school systems in the fall means at-risk children may not get screenings or preventive treatments from in-school clinics (Cramer 2020).

Experts are concerned how this current pandemic has made a bad situation even worse. For instance, James Crall, chair of the Division of Public Health and 
Community Dentistry at the University of California-Los Angeles School of Dentistry, echoes this view when he states that "There were already some underlying trends that I think COVID-19 is going to exacerbate" (Williams 2020).

These disparities are a result of deep systemic issues. The supply of dentists in this country reflects a number of historic factors, such as underrepresentation of people of color entering the field. Less than $4 \%$ of dentists in the US are Black (ADA 2015). If the high cost of obtaining a dental education is one significant barrier, then one possible piece of the solution is to reduce the debt load that students incur (Durbin and Rubio 2020). Moreover, the supply of dental professionals is disproportionately distributed among higher SES communities. Social determinants of health, such as where one lives, employment status, and educational background, all have a profound impact upon health outcomes. Oral health outcomes are no different. Patients who have private insurance through an employer tend to have the best dental coverage and hence the best access to dental care. Patients who lack such insurance may not even have dental coverage. Often, dental care is viewed as a luxury instead of a necessity.

While much of what has been seen during the pandemic regarding access to dental care has been bleak, some developments may actually improve access to care for patients and better integrate dentists into primary care. For example, acceptance of teledentistry is emerging and with it better integration of electronic health records between dentistry and medicine (Simon 2020). Additionally, "[a] s COVID-19 testing capabilities expand, dental offices may become a convenient site for walk-in testing..." which will only enhance the recognition of dentists as primary care providers and potentially pave the way to increase the scope of dental practice to include vaccinations (Simon 2020). Currently only two states allow dentists to administer vaccines, with a third law due to become effective this year (Brian 2020).

As some commentators have stated, "Dental care, particularly preventive dental care, is an extremely important determinant of oral health inequality" (Patrick et al. 2006). The current situation has also highlighted that oral health is part of overall health and no one should be forced to choose between receiving oral health or any other primary health care.

\section{Conclusion}

The current pandemic has revealed deep-seated disparities in health care in general and in oral health care specifically. Despite this unjust history, we have the ability to re-shape our health care system to better meet the needs of all people. For too long, oral health care has been segregated from medical care. The pandemic has shed light on why and how such an approach is detrimental to overall health. Instead of viewing oral health care as somehow different from medical care, recognizing the central importance of oral health as a part of primary health care is critical. Although the current pandemic has brought much suffering to millions of people, it also offers an opportunity to reshape how health care is 
financed and delivered, especially oral health care. Hopefully, the slogan of this pandemic: "We are all in this together" will be realized.

\section{References}

Agency for Healthcare Research and Quality (2020). COVID-19 and dentistry: Challenges and opportunities for providing safe care https://psnet.ahrq.gov/primer/covid-19-and-dentistry-challenges-andopportunities-providing-safe-care

American Dental Association (ADA) (2015). The dentist workforce-key facts (Pamphlet). https://www. ada.org/ /media/ADA/Science\%20and\%20Research/HPI/Files/HPIgraphic_0716_1.pdf?la=en

American Dental Association (ADA) (2020). Current policies. https://www.ada.org/en/advocacy/currentpolicies

American Dental Association News (ADANews) (2020). ADA recommending dentists postpone elective procedures. https://www.ada.org/en/publications/ada-news/2020-archive/march/ada-recommendingdentists-postpone-elective-procedures

Berger, D. (2020). HPI: 99\% of dental offices were open mid-July. ADA News Webinar: https://www.ada. org/en/publications/ada-news/2020-archive/august/ada-airs-on-demand-webinar-on-latest-data-onrecovery

Brian, Z. (2020). Dentists' role in vaccination: An opportunity for public health impact. NC Oral Health Collaborative. https://oralhealthnc.org/dentists-role-in-vaccination-an-opportunity-for-public-healt h-impact/

Brian, Z., Weintraub, J.A. (2020). Oral health and COVID-19: Increasing the need for prevention and access. Preventing chronic disease. 17. https://www.cdc.gov/pcd/issues/2020/20_0266.htm

Centers for Disease Control (CDC) (2020). Guidance for dental settings: Interim infection prevention and control guidance for dental settings during the Coronavirus Disease 2019 (COVID-19) pandemic. https://www.cdc.gov/coronavirus/2019-ncov/hcp/dental-settings.html\#section-1

Cramer, M. (2020). School closures cut a critical line to dental care for poor students. New York Times. https://www.nytimes.com/2020/09/14/us/school-closings-children-dental-care.html

Durbin, R and Rubio, M (2020). How can we fix our health worker shortage? Start with tackling student debt. Roll call. https://www.rollcall.com/2020/08/06/how-can-we-fix-our-health-worker-shortagestart-with-tackling-student-debt/

Evans, M. K. (2020). Covid's color line-infectious disease, inequity, and racial justice. New England Journal of Medicine. https://doi.org/10.1056/NEJMp2019445.

FAIR Health, Inc. (2020). Dental services and the impact of COVID-19: An analysis of private claims. New York, NY: FAIR Health, Inc. https://s3.amazonaws.com/media2.fairhealth.org/brief/asset /Dental\%20Services\%20and\%20the\%20Impact\%20of\%20COVID-19\%20-\%20An\%20Analysis \%20of\%20Private\%20Claims $\% 20-\% 20$ A $\% 20$ FAIR\%20Health\%20Brief.pdf

Galewitz, P. (2020). Reopening dental offices for routine care amid pandemic touches a nerve. Kaiser Health News. https://khn.org/news/reopening-dental-offices-for-routine-care-amid-pandemic-touch es-a-nerve/

Gantz, S. (2020). Pennsylvania adjusts dental restrictions to allow emergency procedures. The Philadelphia Inquirer. https://www.inquirer.com/health/coronavirus/coronavirus-covid19-dental-emergency20200327.html

Guggenheimer, P. (2020). State health department reverses order shutting down dentists during COVID19 crisis. Trib Live. https://triblive.com/local/pittsburgh-allegheny/state-health-department-reversesorder-shutting-down-dentists-during-covid-19-crisis/

Guggenheimer, P. (2020). WHO walks back advice to delay routine dental work, blames media. Trib Live. https://triblive.com/local/regional/who-walks-back-advice-to-put-off-routine-dental-work-blame s-media/

Kalenderian, E., Xiao, Y., Spallek, H., Franklin, A., Olsen, G., Walji, M. (2020). COVID-19 and dentistry: Challenges and opportunities for providing safe care. Patient Safety Network. https://psnet .ahrq.gov/primer/covid-19-and-dentistry-challenges-and-opportunities-providing-safe-care

Koop, C.E. (2000). Oral health in America: A report of the surgeon general. Rockville, Md.; U.S. Department of Health and Human Services, National Institute of Dental and Craniofacial Research, National Institutes of Health. 
Mervosh, S., Lu, D., Swales, V. (2020) See which states and cities have told residents to stay home. The New York Times. https://www.nytimes.com/interactive/2020/us/coronavirus-stay-at-home-order.html

Patrick, D. L., Lee, R. S. L., Nucci, M., Grembowski, D., Jolles, C. Z., \& Milgrom, P. (2006). Reducing oral health disparities: A focus on social and cultural determinants. BMC Oral Health., 6(1), S4.

Pritzker, J.B. (2020). Executive order in response to COVID-19 (Illinois). https://www2.illinois.gov/ IISNews/21288-Gov._Pritzker_Stay_at_Home_Order.pdf

Simon, L. (2020). How will dentistry respond to the Coronavirus Disease 2019 (COVID-19) pandemic. JAMA Health Forum. https://jamanetwork.com/channels/health-forum/fullarticle/2766388

Sydney Policy Lab (2020). COVID-19 and the dental profession: Professional tensions and ethical quandaries. The University of Sydney. https://www.sydney.edu.au/content/dam/corporate/documents/ sydney-policy-lab/policy-paper_covid-19-and-the-dental-profession.pdf

U.S. Department of Health and Human Services (2000). Oral health in America: A report of the surgeon general. Rockville, Md., U.S. Department of Health and Human Services, National Institute of Dental and Craniofacial Research, National Institutes of Health.

U.S. Department of Health and Human Service. (2010). Healthy people with understanding and improving health and objectives for improving health. Washington: U.S. Government Printing Office.

Williams, J.P. (2020). Pandemic threatens to deepen disparities in dental care. US News. https://www. usnews.com/news/healthiest-communities/articles/2020-08-04/coronavirus-threatens-to-deepe n-racial-disparities-in-dental-health

World Health Organization (WHO) (2020). Considerations for the provision of essential oral health services in the context of COVID-19. https://www.who.int/publications/i/item/who-2019-nCoV-oralhealth-2020.1

Publisher's Note Springer Nature remains neutral with regard to jurisdictional claims in published maps and institutional affiliations. 